\title{
Asymptotic Analysis of a Viscous Thread Extending Under Gravity
}

\author{
Jonathan J. Wylie ${ }^{\mathrm{a}, \mathrm{b}}$, Huaxiong Huang ${ }^{\mathrm{c}, \mathrm{b}}$, Robert M. Miura ${ }^{\mathrm{d}, \mathrm{b}}$ \\ ${ }^{a}$ Department of Mathematics, City University of Hong Kong, Tat Chee Avenue, Kowloon, Hong Kong. \\ ${ }^{b}$ Center for applied Mathematics and Statistics, New Jersey Institute of Technology, Newark, NJ 07102, U.S.A.. \\ ${ }^{c}$ Department Mathematics and Statistics, York University, Toronto, Ontario, Canada M3J 1P3. \\ ${ }^{d}$ Department of Mathematical Sciences, New Jersey Institute of Technology, Newark, NJ 07102 USA.
}

\begin{abstract}
Despite extensive research on extensional flows, there is no complete explanation for why highly viscous fluids extending under gravity can form such persistent and stable filaments with no sign of destabilization from surface tension. We therefore investigate the motion of a slender axisymmetric viscous thread that is supported at its top by a fixed horizontal surface and extends downward under gravity. In the case in which inertia and surface tension are initially negligible, we consider the long-wavelength equations for the full initial-boundary-value problem for a thread of arbitrary initial shape. We show that, eventually, the accelerations in the thread become sufficiently large that the inertial terms become important. Thus, we keep the inertial terms and, using matched asymptotic expansions, obtain solutions for the full initialboundary-value problem. We show that the dynamics can be divided into two generic cases that exhibit very different behaviour. In the first case, the thread develops a long thin region that joins together two fluid masses. In this case, we use order-of-magnitude estimates to show that surface-tension-driven pinching will not occur if the square root of the Reynolds number is much greater than the initial aspect ratio divided by the Bond number. In the second case, the thread becomes thin near the horizontal surface. In this case, we show that the long-wavelength equations will ultimately break down and discuss the role of inertia in determining the dynamics. The asymptotic procedures require a number of novel techniques and the resulting solutions exhibit surprisingly rich behavior. The solution allows us to understand the mechanisms that underlie highly persistent filaments.
\end{abstract}

Keywords: Viscous threads, extensional flows, gravity-driven flows

\section{Introduction}

The analysis of flows of filaments represents one of the most important problems in classical fluid mechanics. Work on this subject dates back over 100 years to the pioneers of fluid mechanics such as Rayleigh (1878) and Trouton (1906). A particularly natural problem is that of a thread of highly viscous fluid attached to a horizontal surface from below and extending under gravity. Although this problem has received significant attention (Wilson 1988, Kaye 1991, Stokes et al. 2000, Huang et al. 2003, Huang et al. 2007, Bradshaw-Hajek et al. 2007, Stokes et al. 2011), there are still a number of outstanding fundamental questions about such flows. Some of these questions are highlighted in a recent review article by Eggers \& Villermaux (2008). They describe the substantial progress and the sometimes remarkable agreement between theory and experiments for surface-tension-driven pinching of thin filaments at high and moderate Reynolds numbers. However, in a subsection entitled 'Honey, why are you so thin?', they discuss experiments in which very viscous threads extending under gravity form extraordinarily long filaments with no sign of destabilization from surface tension. A similar phenomenon can occur in the fabrication of glass

Email addresses: mawylie@cityu.edu.hk (Jonathan J. Wylie), hhuang@yorku.ca (Huaxiong Huang), miura@njit.edu (Robert M. Miura)

Preprint submitted to Physica D

July 25, 2015

(C) 2015. This manuscript version is made available under the Elsevier user license http://www.elsevier.com/open-access/userlicense/1.0/ 
microelectrodes (Huang et al. 2003, Huang et al. 2007). Here, a long thin glass tube is hung from a support and a weight is attached to the bottom. The glass is then heated so that the viscosity of the glass decreases and relatively rapid extension occurs. If fracturing of the glass does not occur (e.g. if the glass temperature is sufficiently high), this process can also lead to the formation of extremely long thin filaments.

Indeed, the question of whether these threads can extend indefinitely in the absence of noise remains an open question. In this paper, we will address the need for a quantitative theory by developing an asymptotic theory that can solve the full initial-boundary-value problem for a thread with arbitrary initial shape.

We now briefly review the previous work related to the current problem. A number of authors have considered the problem in which inertia is completely neglected. Wilson (1988) used a long-wavelength theory to consider the extrusion of a viscous fluid from a narrow vertical tube in the presence of gravity. His theory, that neglects inertia, predicts that infinite accelerations occur at a finite time, thus violating the validity of neglecting inertia. Stokes et al. (2000) considered the extension under gravity of a mass of viscous fluid attached to the underside of a horizontal boundary. They considered both long-wavelength theory and finite element simulations and showed that, in the absence of inertia, infinite accelerations will also occur. Stokes et al. (2011) included the effects of surface tension for a thread extending under gravity and paid particular attention to the role played by surface tension near the bottom end of the thread. $\mathrm{Al}$ Khatib (2003) used long-wavelength theory to study a viscoplastic thread extending under gravity. Huang et al. (2003) considered the stretching of glass tubes under gravity with externally applied heating that significantly affects the viscosity of the fluid. Huang et al. (2007) generalized this approach to different types of extensional forces and derived explicit solutions that could be used to identify the factors that control the final shape of glass microelectrodes. Kaye (1991) considered extensional flows with inertia, but did not discuss the role of inertia in cases in which the accelerations become large.

Although the above papers represent significant advances in understanding extensional flows, none of them addresses the mechanism by which inertia ultimately must become important. Wilson (1988) argued that inertia will prevent the infinite accelerations that occur if inertia is completely neglected. He also argued that the minimum cross-sectional area will tend to zero as time tends to infinity if surface tension is completely neglected. However, inertial effects were not the main focus of Wilson's paper and the majority of the analysis is for the zero inertia case. The role of inertia in preventing infinite accelerations is also carefully discussed in Eggers \& Villermaux (2008). In order to further address the question of inertial effects, Stokes \& Tuck (2004) used numerical methods to solve the full initial-boundary-value problem with inertia. They obtained numerical solutions of the long-wavelength equations and compared them with finite element simulations of the full Navier-Stokes equations for zero surface tension. They showed that the thread extends indefinitely, but that computing accurate numerical solutions is very challenging due to large gradients that develop in the solution. Bradshaw-Hajek et al. (2007) developed a more accurate numerical method for the long-wavelength equations and discussed the various difficulties in computing accurate solutions for large times. In addition, these numerical difficulties make determining when and if surface tension effects become important an extremely challenging proposition. In light of these numerical difficulties, an asymptotic solution to this problem would certainly be valuable and this provides one of the major motivations of this paper. We also note that Balmforth et al. (2010) obtained numerical solutions for the dripping of a viscoplastic thread with inertial effects and found good agreement with laboratory experiments.

In fact, despite the extensive numerical work that has been done on this problem, there has been little analytical progress when inertial effects are included. In a previous paper, Wylie et al. (2011) derived asymptotic solutions for the problem of a viscous thread that is pulled at its ends by an imposed fixed force. They showed that the solutions contain complicated internal layers. In this paper, we use an analogous approach and show that internal layers similar to those obtained by Wylie et al. (2011) can occur. In addition, we show that the solutions for a thread extending under gravity exhibit new types of internal and boundary layers that were not present in the previous paper. These new layers require a number of novel asymptotic techniques and the structure of the solution can be surprisingly complicated. We use this solution to explain the mechanism that underlies the persistence and stability of long viscous threads.

In Section 2, we formulate the long-wavelength theory and derive a particularly convenient Lagrangian form of the governing equations. In Section 3, we completely neglect inertia and determine the explicit 
solution. We show that inertia must ultimately become important in one of two different ways. In the first case, inertia first becomes important at a location away from the boundary. In the second case, inertia first becomes important at the boundary. In Sections 4 and 5, we include the effects of inertia and determine the asymptotic solution for the first and second cases, respectively. Finally, we summarize our results and discuss the role of surface tension in Section 6 .

\section{Formulation}

We consider a slender axisymmetric viscous thread that is suspended from a horizontal upper surface (which we will refer to as the 'boundary') and extends vertically downwards due to gravity (see Figure 1). We assume that the thread is composed of an incompressible Newtonian fluid with density $\rho$, surface tension coefficient $\gamma$, and viscosity $\mu$. We denote time by $t$, the vertical distance measured downwards from the boundary by $x$, the cross-sectional area of the thread by $S(x, t)$, the initial cross-sectional area by $S_{0}(x)$, the initial length of the thread by $L$, and the gravitational acceleration by $g$. We define the aspect ratio to be $\delta=\sqrt{\left\langle S_{0}\right\rangle} / L$, where

$$
\left\langle S_{0}\right\rangle=\frac{1}{L} \int_{0}^{L} S_{0}(x) \mathrm{d} x .
$$

\subsection{Eulerian Formulation}

At leading-order in the aspect ratio, $\delta \ll 1$, it has been shown (Fitt et al. 2001) that the axial velocity of the fluid, $U(x, t)$, is independent of the radial coordinate. Using a nondimensionalisation given by

$$
U=\frac{\rho g L^{2}}{3 \mu} \bar{U}, \quad S=\left\langle S_{0}\right\rangle \bar{S}, \quad S_{0}=\left\langle S_{0}\right\rangle \bar{S}_{0}, \quad x=L \bar{x}, \text { and } t=\frac{3 \mu}{\rho g L} \bar{t},
$$

and dropping the overbars, the governing equations (Fitt et al. 2001, Forest et al. 2000, and Yin \& Jaluria 2000) for conservation of mass and momentum, respectively, take the form

$$
\begin{aligned}
S_{t}+(U S)_{x} & =0 \\
R\left(U_{t}+U U_{x}\right) & =\frac{1}{S}\left(S U_{x}+\lambda S^{1 / 2}\right)_{x}+1
\end{aligned}
$$

where

$$
R=\frac{\rho^{2} g L^{3}}{9 \mu^{2}} \text { and } \lambda=\frac{\sqrt{\pi} \gamma}{\rho g L \sqrt{\left\langle S_{0}\right\rangle}} .
$$

We note that equations of this type date back to at least Green (1976).

The dimensionless quantity $R$ is the Reynolds number based on the initial length of the thread and the initial velocity scale that balances the viscous force and gravity. The dimensionless quantity $\lambda$ is a measure of the surface tension force relative to the gravitational force and can be rewritten in terms of the aspect ratio and the Bond number, $B$, as,

$$
\lambda=\frac{\sqrt{\pi} \delta}{B} \quad \text { where } \quad B=\frac{\rho g\left\langle S_{0}\right\rangle}{\gamma} .
$$

In this paper, we will focus on threads that satisfy $\delta \ll 1, R \ll 1$, and $\lambda \ll 1$. Different applications have viscosity scales that vary over many orders of magnitude, from $10^{-2} \mathrm{~kg} / \mathrm{m} / \mathrm{s}$ for water to values in excess of $10^{8} \mathrm{~kg} / \mathrm{m} / \mathrm{s}$ for glass at temperatures around $500^{\circ} \mathrm{C}$. On the other hand, most fluids have surface tension coefficient of order $\gamma \sim 10^{-2}-10^{-1} \mathrm{~kg} / \mathrm{s}^{2}$ and density of order $\rho \sim 10^{3} \mathrm{~kg} / \mathrm{m}^{3}$. Hence, for $\lambda \ll 1$, we will typically require that the product of the length and the radius of the thread is much larger than $10^{-6}-10^{-5} \mathrm{~m}^{2}$. Since $\delta \ll 1$, the condition $\lambda \ll 1$ will automatically be satisfied for threads whose radii are of centimeter scale or larger and will also be satisfied for threads with millimeter-scale radius and tencentimeter-scale lengths. Moreover, to achieve $R \ll 1$, for threads of length of order $L \sim 10^{-1} \mathrm{~m}$, we require 
the viscosity to be of order $\mu \sim 10^{2} \mathrm{~kg} / \mathrm{m} / \mathrm{s}$ (appropriate for syrup) or greater. For threads of length of order $L \sim 1 \mathrm{~m}$, we require the viscosity to be $\mu \sim 10^{4} \mathrm{~kg} / \mathrm{m} / \mathrm{s}$ (appropriate for molten glass at around $900^{\circ} \mathrm{C}$ ) or greater. We note that for molten glass, the viscosity is a very strong function of temperature and depending on the temperature, $R$ can be many orders of magnitude smaller.

The initial conditions for (3) and (4) are given by

$$
U=0 \text { and } S=S_{0}(x) \text { at } t=0 .
$$

For the full Navier-Stokes equations, appropriate conditions are zero normal velocity through the boundary and no-slip. However, since the long-wavelength equations represent a singular perturbation of the Navier-Stokes equations, one must drop one of the boundary conditions. It is most natural to keep the zero normal velocity condition

$$
U=0 \quad \text { at } \quad x=0 .
$$

With this boundary condition, one can readily observe (cf. Figures 3 and 5 of Stokes and Tuck, 2004) that there is a boundary layer of width $\delta$ that is present in the solution of the Navier-Stokes equations that is not present in the solution to the long-wavelength equations. In this boundary layer, the velocity is dependent on the radial coordinate and the long-wavelength equations do not hold. We note, therefore, that solutions of the long-wavelength equations will only be strictly valid for $x \gg \delta$. We also note that there are some additional subtleties associated with the condition at the boundary for the long-wavelength equations that we will discuss in detail in Section 6 .

At the bottom of the thread, zero stress is assumed and so the boundary condition is

$$
S U_{x}+\lambda S^{1 / 2}=0 \quad \text { at } \quad x=\ell(t)
$$

where $\ell(t)$ is the dimensionless location of the bottom of the thread at time $t$. The function $\ell(t)$ satisfies the ordinary differential equation that corresponds to the kinematic condition at the bottom of the thread,

$$
\frac{\mathrm{d} \ell}{\mathrm{d} t}=U(\ell(t), t)
$$

subject to the initial condition, $\ell(0)=1$. Strictly speaking, in the case $S_{0}(1)=0$, the long-wavelength equations break down in a small region close to the bottom of the thread and surface tension is the dominant force. However, as we will explain in Section 6, the time required by surface tension to significantly alter the shape of the thread greatly exceeds the time for which our theory remains valid. Moreover, even though surface tension can drive shape adjustments near the end of the thread, for $\lambda \ll 1$, these adjustments do not affect the dynamics higher up the thread (Balmforth et al. 2010). A detailed discussion of the boundary condition at the bottom of the thread can also be found in Stokes et al. (2011).

\subsection{Lagrangian Formulation}

Although there are a number of important differences, the methodology employed in deriving Lagrangian equations is broadly similar to that used by Wylie et al. (2011). For completeness, we briefly describe the derivation process and refer the reader to Wylie et al. (2011) for details. We transform from the Eulerian variables $(x, t)$ to scaled Lagrangian variables $(\xi, \tau)$. We denote $s(\xi, \tau)=S(x, t), s_{0}(\xi)=S_{0}(x)$, and $u(\xi, \tau)=U(x, t)$. We use a scaled Lagrangian framework in which fluid elements travel with the fluid velocity, $U=X_{\tau}$, where $\tau=t$. Here, $X(\xi, \tau)$ is the spatial location of a material element at time $\tau$, and $\xi$ is the Lagrangian variable given by

$$
\xi=\int_{0}^{X(\xi, 0)} S_{0}(\hat{x}) \mathrm{d} \hat{x} \quad \text { at } \quad \tau=0 .
$$

The Lagrangian variable, $\xi$, labels each fluid element in terms of its initial location. For a given fluid element, the label $\xi$ can be viewed as the fraction the mass that is in the portion of the thread between the boundary and the fluid element. The non-dimensionalization (2) we have adopted implies that $\int_{0}^{1} S_{0}(\hat{x}) \mathrm{d} \hat{x}=1$ and 
therefore, the bottom of the thread is located at $\xi=1$. We use (11) rather than the usual Lagrangian variable $(\xi=X(\xi, 0))$ because it gives a particularly simple form of the final equation.

In the new variables, the equations for mass (3) and momentum (4) are given by

$$
\begin{aligned}
s_{\tau} & =-s^{2} u_{\xi} \\
R u_{\tau} & =\left(s^{2} u_{\xi}+\lambda s^{1 / 2}\right)_{\xi}+1
\end{aligned}
$$

When one has obtained the solution $s(\xi, \tau)$, one can compute the physical location $X$ of a material point that was initially labelled by $\xi$ using

$$
X(\xi, \tau)=\int_{0}^{\xi} \frac{\mathrm{d} y}{s(y, \tau)} .
$$

In this paper, we will consider the case in which $R \ll 1$ and $\lambda \ll 1$. It is therefore natural to proceed by setting $R=\lambda=0$. However, the quantities $R$ and $\lambda$ are estimates for the size of the inertial and surface tension terms based on the initial shape of the thread. Stokes \& Tuck (2004) have shown that the thread will ultimately accelerate significantly, at which point, the effect of inertia must become important. We will therefore retain the inertial terms.

The surface tension terms are more subtle. Stokes \& Tuck (2004) argued that surface tension effects may ultimately become important. In the case of a thread extended by a fixed force, Wylie et al. (2011) considered threads that either have a minimum at the pulled end or are symmetric with a minimum in the center. For these cases, they showed that surface tension will remain negligible throughout the entire extensional process. We will begin our analysis by neglecting the surface tension term by setting $\lambda=0$ in (13). We will return to the validity of neglecting this term in Section 6 .

Setting $\lambda=0$ in (13), eliminating the $s^{2} u_{\xi}$ term using (12), integrating with respect to time, and applying the initial conditions $(7)$ yields

$$
R u=\left(s_{0}-s\right)_{\xi}+\tau \text {. }
$$

This equation describes how the momentum of the thread evolves from the initial condition in which $u=0$ and $s=s_{0}$. On the one hand, a given fluid element experiences gravity and the momentum gain associated with this increases linearly with the time $\tau$. On the other hand, there is a momentum associated with stretching the element from its initial shape that gives rise to the $\left(s_{0}-s\right)_{\xi}$ term. Using (15) to eliminate $u$ from (12), we obtain

$$
R s_{\tau}=-s^{2}\left(s_{0}-s\right)_{\xi \xi} .
$$

This equation is similar in form to that derived by Wylie et al. (2011) for the problem of a filament stretched by an applied force in the absence of gravity.

The initial condition (7) is given by

$$
s(\xi, 0)=s_{0}(\xi)
$$

One of the boundary conditions can be obtained from (15) and (8) and is given by

$$
s_{\xi}(0, \tau)=s_{0}^{\prime}(0)+\tau .
$$

Since there is zero velocity at the boundary, this boundary condition states that the momentum associated with stretching the fluid element at the boundary must balance the gravitational term. To obtain the other boundary condition, we note that (9) with $\lambda=0$ implies that $s^{2} u_{\xi}(1, \tau)=0$. Substituting into (12), integrating with respect to time and using the initial condition (17), we obtain

$$
s(1, \tau)=s_{0}(1)
$$

The fluid element at the bottom of the thread has no weight below it and hence will not experience any stretching. Therefore this boundary condition states that the cross-sectional area at the bottom of the thread remains constant. Finally, we note that (16), (17), (18), and (19) form a closed system of equations. 


\section{Zero Inertia Case}

In order to understand how inertia becomes important, we begin by considering the case of zero inertia. We note that this zero inertia problem has been studied by Stokes et al. (2000), who obtained similar results to those we will obtain here. However, with our choice of Lagrangian variables (11), the solutions take a particularly simple form. We proceed by setting $R=0$ in (16), which yields $s^{2}\left(s_{0}-s\right)_{\xi \xi}=0$. This can be solved subject to the boundary conditions (18) and (19) to yield

$$
s(\xi, \tau)=s_{0}(\xi)-\tau(1-\xi) .
$$

Since inertia is negligible, (20) reflects the balance of the momentum associated with stretching and the gravitational term. We note that the amount of deformation $s_{0}-s$ that a fluid element experiences is proportional to the weight of the thread that is below it. This weight is proportional to the dimensionless mass below the fluid element that is given by $1-\xi$. As noted by Stokes et al. (2000), this solution is clearly not uniformly valid in time because the cross-sectional area will become negative at sufficiently large times. The solution will break down when $s(\xi, \tau)$, given by $(20)$, first becomes zero. This occurs at time $\tau_{s}$ and at location $\xi_{s}$, where

$$
\tau_{s}=\min _{\xi} \frac{s_{0}(\xi)}{1-\xi} \quad \text { and } \quad \xi_{s}=\underset{\xi}{\arg \min } \frac{s_{0}(\xi)}{1-\xi} .
$$

These expressions are consistent with the results of Stokes et al. (2000).

In fact, depending on the initial profile, $s_{0}(\xi)$, there are two generic cases in which the solution can break down. In case i the solution breaks down at a point that is bounded away from the boundary (see Figure 2), whereas in case ii the breakdown occurs at the boundary (see Figure 3).

\subsection{Case i: inertialess solution breaks down away from the boundary}

The condition on $s_{0}(\xi)$ for the solution to break down at a point located away from the boundary can be obtained from (20) and is given by

$$
\exists \xi \in(0,1) \quad \text { s.t. } \quad s_{0}(\xi)<(1-\xi) s_{0}(0) .
$$

In order to determine the values of $\xi_{s}$ and $\tau_{s}$, one makes use of the conditions that $s(\xi, \tau)=0$ and $s_{\xi}(\xi, \tau)=0$ when the breakdown occurs to obtain the following two equations

$$
s_{0}\left(\xi_{s}\right)-\tau_{s}\left(1-\xi_{s}\right)=0
$$

and

$$
s_{0}^{\prime}\left(\xi_{s}\right)+\tau_{s}=0 .
$$

In Figure 2, we plot a schematic of the cross-sectional area at various times.

Prior to the breakdown of the solution, the total length of the thread can be computed using (14). As the breakdown time is approached $\left(\tau \rightarrow \tau_{s}\right)$, the integral (14) is dominated by the contribution near $\xi=\xi_{s}$. Expanding (20) near $\xi=\xi_{s}$ and using (23) and (24), we obtain

$$
X(1, \tau) \sim \int_{0}^{1} \frac{\mathrm{d} \xi}{\left(\tau_{s}-\tau\right)\left(1-\xi_{s}\right)+\frac{1}{2} s_{0}^{\prime \prime}\left(\xi_{s}\right)\left(\xi-\xi_{s}\right)^{2}+\left(\xi-\xi_{s}\right)\left(\tau-\tau_{s}\right)} \quad \text { as } \quad \tau \rightarrow \tau_{s}^{-} .
$$

On the right-hand side of the above expression, we have assumed the generic case in which $s_{0}^{\prime \prime}\left(\xi_{s}\right)>0$. A straightforward calculation shows that the dominant contribution to the integral comes from the region $\xi-\xi_{s}=O\left(\left(\tau_{s}-\tau\right)^{1 / 2}\right)$, and we obtain

$$
X(1, \tau) \sim \pi \sqrt{\frac{2}{s_{0}^{\prime \prime}\left(\xi_{s}\right)\left(\tau_{s}-\tau\right)\left(1-\xi_{s}\right)}} \quad \text { as } \quad \tau \rightarrow \tau_{s}^{-} .
$$

This implies that the extension tends to infinity in a finite time and hence the acceleration must also tend to infinity. This clearly demonstrates that the neglect of the inertial terms is not valid for $\tau \geq \tau_{s}$. We note that a similar type of asymptotic behaviour has been obtained for the stretching of glass sheets (Wilmott 1989). 


\subsection{Case ii: inertialess solution breaks down at the boundary}

The condition on $s_{0}(\xi)$ for the solution to break down at the boundary is given by

$$
s_{0}(\xi)>(1-\xi) s_{0}(0) \quad \forall \xi \in(0,1) .
$$

In this case, $\xi_{s}=0$ and $\tau_{s}=s_{0}(0)$. We illustrate this in Figure 3. As in case i, the total length of the thread is given by (14) and (20). Expanding $s_{0}(\xi)$ near $\xi=0$, we can rewrite $s_{0}(\xi)-\tau(1-\xi)=$ $s_{0}(0)-\tau+\left[s_{0}^{\prime}(0)+\tau\right] \xi+O\left(\xi^{2}\right)$. As $\tau \rightarrow s_{0}(0)$, the integral (14) is dominated by the contribution near $\xi=0$. In the generic case in which $s_{0}^{\prime}(0)>-s_{0}(0)$, we obtain

$$
X(1, \tau) \sim-\frac{\ln \left[s_{0}(0)-\tau\right]}{s_{0}^{\prime}(0)+s_{0}(0)} \quad \text { as } \quad \tau \rightarrow s_{0}(0)^{-} .
$$

Similar to case $\mathrm{i}$, this indicates that the neglect of the inertial terms is not valid for $\tau \geq s_{0}(0)$. This generalizes the result of Stokes et al. (2000) who obtained the extension for a cylindrical thread with initially constant cross-section.

In the following sections, we will show that inertia regularizes both of these two cases. The two cases exhibit very different dynamics and require completely different asymptotic techniques.

\section{Inertial Effects Away from the Boundary: Case i}

In this section, we derive asymptotic solutions for the case in which the thread first becomes thin at a point that is located away from the boundary.

The basic methodology to solve this problem is similar to that used by Wylie et al. (2011) for a thread pulled at its end by a fixed force. However, in the current problem, the solution structure is more complicated and transition layers arise that were not considered in Wylie et al. (2011), as shown below.

If $R \ll 1$, the main idea is to divide the thread into regions in which $s=O(1)$ and regions in which $s$ is small. For regions in which $s=O(1)$, the inertial term in (16) is negligible. Thus, to leading order, we must solve $s^{2}\left(s-s_{0}\right)_{\xi \xi}=0$. Since $s=\mathrm{O}(1), s_{0}-s$ must be a linear function of $\xi$. We will refer to these regions as "outer regions".

On the other hand, for regions in which $s$ is small, $s_{\xi \xi}$ will, generically, be much smaller than $s_{0}^{\prime \prime}$. Therefore, at leading order, (16) can be written as

$$
R\left(\frac{1}{s}\right)_{\tau}=s_{0}^{\prime \prime}(\xi) .
$$

This can be integrated with respect to time to give

$$
s=\frac{R}{s_{0}^{\prime \prime}(\xi) \tau+E(\xi)}
$$

where $E(\xi)$ is a function of $\xi$ that arises from the integration and must be determined by matching. In particular, we note that $s=O(R)$ in these regions and we will refer to these regions as "necking regions". In this case, inertia, viscous stresses and gravity are all important, however, (29) is simpler than the full equation (16). This is because the thread has become sufficiently thin that the momentum associated with the viscous stretching of a fluid element from its initial shape that gives rise to the $\left(s_{0}-s\right)_{\xi}$ term in (15) has, to leading order, achieved its largest possible value of $s_{0}^{\prime}$. Physically, this means that the thread has become so thin that any further extension will have a negligible contribution to the total momentum needed for stretching the fluid element from its initial shape. The gravitational term in (15) continues to increase with time and this increase can only be balanced by the inertial term. This implies that this portion of the thread is essentially falling under gravity and thinning to conserve mass.

We now show how to determine the locations of the necking and outer regions. As we showed above, $s_{0}-s$ is a linear function of $\xi$ in the outer regions and so we consider the variable $d(\xi, \tau)=s(\xi, \tau)-s_{0}(\xi)$ 
that represents the deviation from the initial profile. In the outer regions, $d$ is a linear function of $\xi$, whereas in the necking regions, $s=O(R)$ and so to leading order $d=-s_{0}(\xi)$. Hence, it is apparent that this problem can be viewed as a simple obstacle problem (Rodrigues 1987) with an elastic string (whose end moves with time) wrapping around an object whose shape is given by $d=-s_{0}(\xi)$ (see Figure 4 ). The necking regions correspond to the elastic string being in contact with the object, whereas the outer regions correspond to the elastic string not being in contact with the object and hence being composed of straight lines in each outer region. The conditions to determine the locations of the end of the string can be determined from (18) and (19). These conditions are a slope condition that varies with time at the boundary, given by $d_{\xi}(0, \tau)=\tau$, and a constraint on the value at the free end, given by $d(1, \tau)=0$.

We must patch together the two types of solutions to construct the complete solution. At the interface between a necking region and an outer region, the flux, $\left(s_{0}-s\right)_{\xi}$ should be continuous and, hence, $d$ and $d_{\xi}$ should be continuous at the interface. These conditions are also the same as those for the standard obstacle problem. Since the standard obstacle problem can be solved using a simple geometrical method, the problem of determining the leading-order locations of the outer and necking regions can also be solved by purely geometrical methods. We note that only the boundary condition at $\xi=0$ is time-dependent. Therefore, if there is more than one outer region, then only the outer region connecting $\xi=0$ to the object will be time-dependent and all other outer regions will be stationary. This implies that only the contact point nearest to $\xi=0$ will move and the other contact points will remain stationary. An illustration of the original and the obstacle formulations is given in Figure 4.

For simplicity, we now consider the case in which there is only one necking region. Cases with multiple necking regions can be considered using a similar approach. We define the location of the edge of the necking region closest to $\xi=0$ as $\xi_{m}(\tau)$ where the subscript $m$ represents 'moving'. The location of the other edge of the necking region does not move with time. Therefore, its value is determined when the necking layer first appears at a time $\tau_{s}$ and is given by $\xi_{s}$, which was obtained in Section 3 by solving (23) and (24). In Figure 5, we plot a schematic of the different regions of the $(\xi, \tau)$ space. We note that, the subscript $s$ represents 'stationary' since (in Lagrangian coordinates) this point does not move with time. We also note that for $\tau>\tau_{s}$, the outer solution furthest from the boundary is stationary and is given by

$$
s(\xi, \tau)=s_{0}(\xi)-\tau_{s}(1-\xi) \quad \text { for } \quad \xi>\xi_{s} .
$$

Using (23) and (24), this can be rewritten in the form

$$
s(\xi, \tau)=s_{0}(\xi)-s_{0}\left(\xi_{s}\right)-s_{0}^{\prime}\left(\xi_{s}\right)\left(\xi-\xi_{s}\right) \quad \text { for } \quad \xi>\xi_{s} .
$$

In order to determine the solution in the region $0 \leq \xi<\xi_{m}(\tau)$ and the location of the moving contact point, $\xi_{m}(\tau)$, we match the values and slopes of the necking and outer solutions. In the outer region, $0 \leq \xi<\xi_{m}(\tau), s-s_{0}$ is a linear function that must satisfy (18) and the value and slope conditions $s\left(\xi_{m}, \tau\right)=0$ and $s_{\xi}\left(\xi_{m}, \tau\right)=0$. Hence, the solution is given by

$$
s(\xi, \tau)=s_{0}(\xi)-s_{0}\left(\xi_{m}(\tau)\right)+\tau\left(\xi-\xi_{m}(\tau)\right) \quad \text { for } \quad \xi<\xi_{m}(\tau)
$$

where $\xi_{m}(\tau)$ is determined from

$$
s_{0}^{\prime}\left(\xi_{m}(\tau)\right)+\tau=0
$$

We immediately see that the contact point will continue to move until the thread becomes thin at $\xi=0$. Beyond this time, the thread will undergo thinning similar to case ii that we will describe in Section 5 .

In the vicinity of $\xi_{m}(\tau)$ and $\xi_{s}$, there are transition layers that join the solutions in the necking and outer regions. The transition layer in the vicinity of $\xi_{m}(\tau)$ can be analysed using a similar approach to that used in Wylie et al. (2011), and therefore, we only give a brief description of the result. On the other hand, the transition layer in the vicinity of $\xi_{s}$ was not considered in Wylie et al. (2011) and has a different structure. Therefore, we will analyse it in detail.

We begin with the transition layer in the vicinity of $\xi_{m}(\tau)$. We introduce the following new variables

$$
\chi=\frac{\xi-\xi_{m}(\tau)}{R^{1 / 3} K(\tau)} \quad \text { and } \quad s=\frac{R^{2 / 3}}{M(\tau)} G(\chi)
$$


where $K$ and $M$ are functions of $\tau$ that are chosen to simplify the resulting differential equation and are given by

$$
K=2^{2 / 3}\left[\dot{\xi}_{m}(\tau)\right]^{1 / 3}\left[s_{0}^{\prime \prime}\left(\xi_{m}(\tau)\right)\right]^{-2 / 3} \quad \text { and } \quad M=2^{-1 / 3}\left[\dot{\xi}_{m}(\tau)\right]^{-2 / 3}\left[s_{0}^{\prime \prime}\left(\xi_{m}(\tau)\right)\right]^{1 / 3} .
$$

Substituting into (16) and taking the leading-order terms in $R$, we obtain

$$
G_{\chi}=G^{2}\left(2-G_{\chi \chi}\right)
$$

In this layer, all of the terms in (16) play a role, but due to the thinness of the layer, the $s_{0}^{\prime \prime}$ term is constant to leading order. Dividing by $G^{2}$ and integrating gives

$$
G_{\chi}=2\left(\chi-D_{1}\right)+\frac{1}{G}
$$

where $D_{1}$ is a constant that arises from the integration. Then, (38) can be solved in terms of Airy functions and after matching with the necking region (see Wylie et al. 2011 for details), we obtain

$$
G=\left(\chi-D_{1}\right)^{2}-F^{-1}\left(\chi-D_{1}\right)
$$

where $F(x)=\operatorname{Ai}^{\prime}(x) / \operatorname{Ai}(x)$ and the inverse function $F^{-1}(x)$ selects the root in the range $\left(Z_{0}, \infty\right)$ where $Z_{0}=-2.338 \cdots$ is the first zero of the Airy function. Matching also requires that the function $E(\xi)$ in (30) be given by $E(\xi)=-s_{0}^{\prime \prime}(\xi) \tau_{m}(\xi)$ where $\tau_{m}(\xi)$ is the inverse function of $\xi_{m}(\tau)$. Using (24), we see that $\tau_{m}(\xi)=-s_{0}^{\prime}(\xi)$, and hence the solution in the necking region is given by

$$
s=\frac{R}{s_{0}^{\prime \prime}(\xi)\left[\tau+s_{0}^{\prime}(\xi)\right]} .
$$

We note that (39) contains an undetermined constant $D_{1}$. Using leading-order matching, this constant cannot be determined. However, the matching conditions apply at $\chi \rightarrow \pm \infty$ and the equation (37) is invariant under translations in $\chi$. Different values of $D_{1}$ thus correspond to small translations in the location of the transition layer. We can therefore arbitrarily set $D_{1}=0$. We note that the solution in the necking region (40) is completely determined by matching with the moving transition layer in the vicinity of $\xi_{m}(\tau)$ and does not depend on the details of the solution in the stationary transition layer in the vicinity of $\xi_{s}$.

We now turn our attention to the transition layer at $\xi=\xi_{s}$. We require that the solution in the transition layer matches the necking solution (40). Noting that $s_{0}^{\prime}\left(\xi_{s}\right)=-\tau_{s}$ from (24), we see that as $\xi \rightarrow \xi_{s}$, the necking solution takes the form

$$
s=\frac{R}{s_{0}^{\prime \prime}\left(\xi_{s}\right)\left(\tau-\tau_{s}\right)} .
$$

Thus, the scaling behaviour for $s$ is $s \sim R\left(\tau-\tau_{s}\right)^{-1}$. Substituting this into (16), we see that the distinguished limit in which all terms are of comparable order is achieved when $\xi$ scales like $\xi-\xi_{s} \sim R^{1 / 2}\left(\tau-\tau_{s}\right)^{-1 / 2}$. We therefore seek solutions of the form

$$
s=\frac{R}{s_{0}^{\prime \prime}\left(\xi_{s}\right)\left(\tau-\tau_{s}\right)} H(\psi) \quad \text { where } \quad \psi=\frac{\left(\xi-\xi_{s}\right)\left(\tau-\tau_{s}\right)^{1 / 2} s_{0}^{\prime \prime}\left(\xi_{s}\right)}{R^{1 / 2}} .
$$

Substituting (42) into (16) and expanding around $\xi=\xi_{s}$, we obtain the leading-order ordinary differential equation

$$
H-\frac{\psi}{2} H^{\prime}=H^{2}\left(1-H^{\prime \prime}\right)
$$

Similarly to the previous transition layer, in this layer, all of the terms in (16) play a role, but the $s_{0}^{\prime \prime}$ term is constant to leading order. The boundary conditions are

$$
H \rightarrow 1 \text { as } \psi \rightarrow-\infty
$$


which comes from matching with the necking solution (40), and

$$
H \rightarrow \frac{\psi^{2}}{2} \text { as } \psi \rightarrow \infty
$$

which comes from matching with the outer solution (32). Multiplying (43) by $\psi H^{-2}$ and integrating yields

$$
\frac{\psi^{2}}{2 H}-\frac{\psi^{2}}{2}=-\psi H^{\prime}+H-2 D_{2}
$$

where $D_{2}$ is a constant of integration. This can be integrated again and after applying the matching condition (44), the solution can be expressed in terms of Whittaker functions as

$$
4\left(H-2 D_{2}\right) W_{\frac{1}{4}+D_{2}, \frac{1}{4}}\left(\frac{\left(-\psi^{2}-4 D_{2}+2 H\right)^{2}}{4 \psi^{2}}\right)=\psi^{2}\left(1-2 D_{2}\right) W_{-\frac{3}{4}+D_{2}, \frac{1}{4}}\left(\frac{\left(-\psi^{2}-4 D_{2}+2 H\right)^{2}}{4 \psi^{2}}\right) .
$$

In Figure 6, we plot $H(\psi)$ for various values of $D_{2}>1 / 2$. By using properties of the Whittaker function, one can readily see that the boundary condition (45) is automatically satisfied for any value of $D_{2}>1 / 2$. This means that the leading-order matching procedure that gives (43)-(45) cannot uniquely determine the constant $D_{2}$. We note that we encountered a similar issue when solving (37) for the moving transition layer for which the parameter $D_{1}$ could not be determined. However, in that case, changes in $D_{1}$ just represented a simple translation in the location of the transition layer, whereas changes in $D_{2}$ have a more complicated effect on the solution for $H$. To determine $D_{2}$, one would have to perform higherorder matching. Nevertheless, despite the fact that we are unable to uniquely determine the solution using leading-order matching, we can obtain the scaling and useful information about the form of the solution in this transition layer.

Finally, we can determine an asymptotic expression for the total extension of the thread given by (14). It is easy to see that, at leading order, the main contribution to the extension comes from the necking region and that the contributions from the two transition regions are of higher order. Using (40), we obtain

$$
X(1, \tau)=\frac{1}{R} \int_{\xi_{m}(\tau)}^{\xi_{s}} s_{0}^{\prime \prime}(\xi)\left[\tau+s_{0}^{\prime}(\xi)\right] \mathrm{d} \xi .
$$

This can be integrated, and after using (24) and (34), we obtain

$$
X(1, \tau)=\frac{1}{2 R}\left(\tau-\tau_{s}\right)^{2} .
$$

This expression represents a solution in which the fluid in the necking region is sufficiently thin that it exerts a negligible force on the fluid below it. Therefore, the fluid below the necking region experiences motion close to gravitational free fall.

\section{Inertial Effects Near Boundary: Case ii}

If the inertialess solution first breaks down at the boundary, the situation is quite different from case $\mathrm{i}$ and a completely different asymptotic method must be employed. We will consider the general case later, but for simplicity, we begin with the case $s_{0}(\xi) \equiv 1$. In this case, using $(21)$, we see that the small Reynolds number solution (20) is valid for $\tau<1$. Seeking a naive expansion in powers of $R$, to obtain the asymptotic correction we obtain

$$
s=1-\tau+\tau \xi+R\left\{\frac{2}{\tau^{3}} \ln (1-\tau+\tau \xi)+\frac{1-\xi}{\tau^{2}}\left[\frac{2-\tau}{1-\tau}+\ln \left(\frac{1-\tau}{1-\tau+\tau \xi}\right)\right]\right\}+O\left(R^{2}\right) .
$$

This expansion breaks down in the region $\xi=O\left(R^{1 / 2}\right)$ when $1-\tau=O\left(R^{1 / 2}\right)$. We therefore proceed by introducing a scaled time $T$ using $\tau=1+R^{1 / 2} T$ and a boundary layer variable $\xi=R^{1 / 2} \eta$. We denote the 
boundary layer solution by $s(\xi, \tau)=R^{1 / 2} p(\eta, T)$ and the outer solution by $s(\xi, \tau)=P(\xi, T)$. Substituting into (16), (18) and (19) with $s_{0}(\xi) \equiv 1$, the boundary layer and outer equations are given by

$$
\begin{aligned}
& R^{1 / 2} p_{T}=p^{2} p_{\eta \eta} \quad \text { subject to } \quad p_{\eta}=1+R^{1 / 2} T \quad \text { at } \quad \eta=0 \\
& R^{1 / 2} P_{T}=P^{2} P_{\xi \xi} \quad \text { subject to } \quad P=1 \quad \text { at } \quad \xi=1 .
\end{aligned}
$$

We proceed by posing expansions of the form

$$
\begin{aligned}
& p(\eta, T)=p_{0}(\eta, T)+R^{1 / 2} p_{1}(\eta, T)+O(R) \\
& P(\xi, T)=P_{0}(\xi, T)+R^{1 / 2} P_{1}(\xi, T)+O(R)
\end{aligned}
$$

and using a standard asymptotic matching procedure.

To determine the boundary layer solution, we substitute (53) into (51) and integrate twice to obtain

$$
p_{0}(\eta, T)=\sigma(T)+\eta,
$$

where $\sigma(T)$ is a function that must be determined by matching. At the next order, we obtain

$$
p_{1}(\eta, T)=-\frac{\mathrm{d} \sigma}{\mathrm{d} T} \ln (\sigma+\eta)+\left(T+\frac{1}{\sigma} \frac{\mathrm{d} \sigma}{\mathrm{d} T}\right) \eta+C(T)
$$

where $C(T)$ is a function that must be determined by matching.

To determine the outer solution, we substitute (54) into (52), integrate twice and match with (55) to obtain

$$
P_{0}(\xi, T)=\xi
$$

At the next order, we match using (55) and obtain

$$
P_{1}(\xi, T)=\sigma(T)(1-\xi) .
$$

Matching the inner and outer solutions, we obtain a Riccati equation for $\sigma$

$$
\frac{\mathrm{d} \sigma}{\mathrm{d} T}+\sigma^{2}+T \sigma=0
$$

As $T \rightarrow-\infty$, we must recover the behavior of (50) near $\tau=1$ and $\xi=0$, that is $s \sim 1-\tau+\xi$. From (55), we see that this corresponds to the condition $\sigma \rightarrow-T$ as $T \rightarrow-\infty$. The solution is therefore,

$$
\sigma(T)=\sqrt{\frac{2}{\pi}} \frac{e^{-T^{2} / 2}}{\operatorname{erfc}(-T / \sqrt{2})} .
$$

We note that the quantity $R^{1 / 2} \sigma$ represents the minimum cross-sectional area of the thread, $s(0, \tau)$. In fact, the above expression for $\sigma$ is also valid $\tau<1$ and yields $s(0, \tau)=1-\tau$. For $T=O(1), \sigma$ will be $O(1)$ and so the minimum cross-sectional area will be of $O\left(R^{1 / 2}\right)$. However, as $T$ gets large, $\sigma$ and hence the minimum cross-sectional area will become exponentially small. In Figure 7, we plot $s(0, \tau)$ against time for $R=10^{-2}$.

For the asymptotic expansion (53) to remain valid as $T$ becomes large, we require that $p_{0}(\eta, T) \gg$ $R^{1 / 2} p_{1}(\eta, T)$. For $\eta=O(1)$ this is automatically satisfied, but for $\eta$ of the order of $\sigma$ or smaller, we require that $R^{1 / 2} T \ln (\sigma) \ll 1$. Using (60) to eliminate $\sigma$, we obtain the requirement $R^{1 / 2} T^{3} \ll 1$, which corresponds to $T \ll R^{-1 / 6}$ or $\tau-1 \ll R^{1 / 3}$. Therefore, at the time when the asymptotic series breaks down, $\sigma$ will be exponentially small with size of order $\exp \left(-R^{-1 / 3} / 2\right)$.

If $\sigma$ becomes small, the logarithmic term in (56) has different behaviour for $\eta \ll \sigma$ and $\eta \gg \sigma$. It is therefore apparent that the scale $\eta \sim \sigma$ is important in determining the behaviour of the solution in the boundary layer. This corresponds to a scale for which $\xi \sim R^{1 / 2} \sigma$. Recall that our long-wavelength theory 
neglected the layer at the boundary of width $\delta$ that is present in the Navier-Stokes equation and so our long-wavelength solution can only asymptotically represent the Navier-Stokes solution while

$$
R^{1 / 2} \sigma \gg \delta
$$

If $\delta \gg R^{1 / 2}$, or $\delta=O\left(R^{1 / 2}\right)$, the condition (61) will not be satisfied for $\tau-1=O\left(R^{1 / 2}\right)$ and the longwavelength theory breaks down before inertia becomes significant. On the other hand, if $\delta \ll R^{1 / 2}$, the long-wavelength theory will be valid for $\tau-1=O\left(R^{1 / 2}\right)$, but eventually, $\sigma$ will decrease until (61) is violated at which time the long-wavelength theory breaks down.

Combining all of the above results, we can obtain a leading-order uniformly valid expression for $s(\xi, \tau)$

$$
s(\xi, \tau)=\xi+R^{1 / 2}(1-\xi) \sigma+R\left(\sigma+\frac{\tau-1}{R^{1 / 2}}\right) \sigma \ln \left(\frac{\xi+R^{1 / 2} \sigma}{1+R^{1 / 2} \sigma}\right) .
$$

We note that the $\ln \left(\xi+R^{1 / 2} \sigma\right)$ term appears to be a higher-order term, but is important when computing the gradient $s_{\xi}$ for values of $\xi=O\left(R^{1 / 2} \sigma\right)$, and is required to satisfy the boundary condition (18) at $\xi=0$. On the other hand, we do not need to include the function $C$ from (56) since this term is of higher order for all values of $\xi$. We have also included the $\ln \left(1+R^{1 / 2} \sigma\right)$ term (that is higher-order for all $\xi$ ) so that (62) exactly satisfies the boundary condition (19) at $\xi=1$.

The extension of the thread for times $\tau-1=O\left(R^{1 / 2}\right)$ can be computed using (14) and (62). One can readily see that the integral will be dominated by the contribution near $\xi=0$, and is given by

$$
X(1, \tau) \sim-\ln \left(R^{1 / 2} \sigma\right) .
$$

For times $R^{1 / 3} \gg \tau-1 \gg R^{1 / 2}, \sigma$ will be exponentially small and leading-order contribution to the extension becomes

$$
X(1, \tau) \sim \frac{(\tau-1)^{2}}{2 R}
$$

This represents a solution for the extension when the thread is sufficiently thin near the boundary that, as in case $i$, it exerts a negligible force on the fluid below it. In this case, the bulk of the fluid experiences motion close to gravitational free fall.

For the case $s_{0}(\xi) \not \equiv 1$, the above approach generalizes in a straightforward way. Defining $\tau=s_{0}(0)+$ $R^{1 / 2} T$, the inner and outer solutions are given by

$$
\begin{aligned}
& p(\eta, T)=\sigma+m \eta+R^{1 / 2}\left[\frac{s_{0}^{\prime \prime}(0) \eta^{2}}{2}+\left(\frac{1}{m \sigma} \frac{\mathrm{d} \sigma}{\mathrm{d} T}+T\right) \eta-\frac{\ln (\sigma+m \eta)}{m^{2}} \frac{\mathrm{d} \sigma}{\mathrm{d} T}+C(T)\right]+O(R) \\
& P(\xi, T)=\left[s_{0}(\xi)-s_{0}(0)(1-\xi)\right]+R^{1 / 2} \sigma(T)(1-\xi)+O(R),
\end{aligned}
$$

where $m=s_{0}(0)+s_{0}^{\prime}(0)$ and $C(T)$ is a function that must be determined by matching. Matching the inner and outer solutions then yields the following Riccati equation for $\sigma$

$$
\frac{\mathrm{d} \sigma}{\mathrm{d} T}+m\left(\sigma^{2}+T \sigma\right)=0
$$

that has solution

$$
\sigma(T)=\sqrt{\frac{2}{m \pi}} \frac{e^{-m T^{2} / 2}}{\operatorname{erfc}(-T \sqrt{m / 2})} .
$$

A leading-order uniformly valid expression for $s(\xi, \tau)$ is given by

$$
s(\xi, \tau)=\left[s_{0}(\xi)-s_{0}(0)(1-\xi)\right]+R^{1 / 2}(1-\xi) \sigma+R\left(\sigma+\frac{\tau-s_{0}(0)}{R^{1 / 2}}\right) \frac{\sigma}{m} \ln \left(\frac{m \xi+R^{1 / 2} \sigma}{m+R^{1 / 2} \sigma}\right) .
$$




\section{Discussion}

In this paper, we have analysed the full initial-boundary-value problem for a slender thread with arbitrary initial shape in the asymptotic limit of low Reynolds number. We have shown that when inertia is completely neglected, the solution must eventually break down in one of two different ways. In the first case, the thread first becomes thin at a location that is bounded away from the boundary, whereas in the second case, it first becomes thin at the boundary. In both cases, if inertia is completely neglected, the accelerations tend to infinity in a finite time, invalidating the neglect of inertia. We have therefore included the inertial terms and used matched asymptotic expansion techniques for the solutions in both cases. In the first case, we have shown that the solution can be obtained by geometric methods identical to those used in solving the problem of an elastic string wrapping around an obstacle. We have shown that there will typically be a thin necking region that connects two outer regions through two transition regions. In the second case, we have shown that the thread thins near the boundary. In fact, even for only moderately small Reynolds numbers, we have shown that the long-wavelength description of the thread must ultimately cease to be valid.

We now return to the question of the validity of the zero normal velocity condition (8) for the longwavelength equations. As we mentioned above, the full Navier-Stokes equations require two conditions at the boundary. Typically, these would be zero normal velocity and no-slip. However, in the long-wavelength approximation, we were forced to drop one of the boundary conditions, namely the no-slip condition. This gives rise to a boundary layer in which the solution of the Navier-Stokes equation adjusts from the no-slip condition to the radius required by the long-wavelength solution. This boundary layer corresponds to a mass of fluid attached to the boundary that maintains its initial radius at the boundary (due to the no-slip condition). The dimensional height of the layer is of the same order as the radius of the thread at the boundary.

We now address the question of whether this 'reservoir' of fluid can experience sufficiently strong gravitational or surface tension forces to give rise to a significant mass flux at the edge of the boundary layer that would invalidate the zero normal velocity condition applied to the long-wavelength equations. In dimensional terms, the flow driven by gravity of a volume of fluid with dimensional radius $R_{0}$ and height $R_{0}$ has an associated mass flux with order-of-magnitude size $\rho g R_{0}^{4} / \mu$. Expressing this in dimensionless variables we obtain an apparent mass flux into the long-wavelength equations $s u=O\left(\delta^{2}\right)$. Hence, in order to apply the zero normal velocity condition on the the long-wavelength equations, we require that $s \gg \delta^{2}$. For case $\mathrm{i}$, this does not present a problem, since $s=O(1)$ at the boundary. In case ii, $s$ will become small and so there is a potential problem. However, in order for our long-wavelength theory to be unaffected by the boundary layer, we required (61) to hold. This represents a more stringent condition than $s \gg \delta^{2}$. A similar analysis for surface-tension driven flows in the boundary layer gives a mass flux with order-of-magnitude size $\gamma R_{0}^{2} / \mu$. When expressed in dimensionless variables, this corresponds to an apparent mass flux into the long-wavelength equations $s u=O(\lambda \delta)$. Since (61) requires that $s \gg \delta$ and we assume that $\lambda \ll 1$, this must also be small. So, for flows driven by both surface tension and gravity, our application of the zero velocity condition is well-justified.

We close by addressing the question of whether neglecting surface tension (by putting $\lambda=0$ ) is valid. To do this, we will estimate the size of the surface-tension effects using order-of-magnitude estimates that are based on the axial scale arising from the gravitationally driven extension. It is possible that other physical mechanisms, such a noise, could lead to smaller scale features in the thread that could induce surface-tension driven pinching. However, this is a separate issue that we will not address here. It will prove convenient to develop a single equation that contains the surface tension terms. This can be obtained by cross-differentiating (12) and (13) to eliminate terms involving $u$ to obtain

$$
R\left(\frac{s_{\tau}}{s^{2}}\right)_{\tau}=\left(s_{\tau}-\lambda s^{1 / 2}\right)_{\xi \xi} .
$$

We proceed by considering the solutions in each of the regions and checking to see whether the surface tension terms are smaller than the leading-order terms in that region. We note that, since $\lambda \ll 1$, the surface tension can become important only at those times after $\tau_{s}$, when at least part of the thread is thin. 
We begin by considering case $\mathrm{i}$ in which the thread becomes thin at a location away from the boundary. In this case, there are five regions to examine: the outer region attached to the boundary, the transition layer in the vicinity of $\xi_{m}(\tau)$, the necking region, the transition layer in the vicinity of $\xi_{s}$, and the outer region far from the boundary. We now consider each of the regions separately. In the outer region attached to the boundary, the dominant term in (70) is the $s_{\tau}$ term. The solution is given by (33) and hence $s=O(1)$ and $s_{\tau}=O(1)$. Since $\lambda \ll 1$, the $s_{\tau}=O(1)$ term in (70) is always larger that the $\lambda s^{1 / 2}$ term which is of $O(\lambda)$. We conclude that surface tension is negligible in this region. In the transition region in the vicinity of $\xi_{m}(\tau)$, the $s_{\tau}$ and $R\left[s^{-2} s_{\tau}\right]_{\tau}$ terms are of the same order and the solution is given by (39). In this region, $s=O\left(R^{2 / 3}\right)$ and $\partial_{\tau}=O\left(R^{-1 / 3}\right)$. Therefore, $s_{\tau}=O\left(R^{1 / 3}\right)$ and $\lambda s^{1 / 2}=O\left(\lambda R^{1 / 3}\right)$, and hence, $s_{\tau} \gg \lambda s^{1 / 2}$ and again surface tension is negligible. In the necking region, the dominant term is $R\left(s^{-2} s_{\tau}\right)_{\tau}$. The solution is given by (40) and $s=O(R), \partial_{\xi}=O(1)$, and $\partial_{\tau}=O(1)$. Therefore,

$$
R\left(\frac{s_{\tau}}{s^{2}}\right)_{\tau}=O(1) \quad \text { and } \quad\left(\lambda s^{1 / 2}\right)_{\xi \xi}=O\left(\lambda R^{1 / 2}\right)
$$

and again the effect of surface tension is negligible. In the transition region in the vicinity of $\xi_{s}$, the $s_{\tau}$ and $R\left[s^{-2} s_{\tau}\right]_{\tau}$ terms are of the same order. The solution is given by $(47), s_{\tau}=O(R), s=O(R)$, and $\lambda s^{1 / 2}=O\left(\lambda R^{1 / 2}\right)$. So, the surface tension terms will only be negligible if $R \gg \lambda R^{1 / 2}$, or equivalently

$$
R \gg \lambda^{2}
$$

Finally, in the outer region far from the boundary, the situation is more subtle. Here, the solution is given by (32) and $s=O(1)$. However, $s_{\tau}$ and $R\left(s^{-2} s_{\tau}\right)_{\tau}$ are identically zero. This implies that surface tension is the dominant force in this region. From a physical viewpoint, this is not surprising since this region represents a large body of fluid that is only attached to the boundary by the thin necking region. It therefore feels very little viscous force from above and hence experiences accelerations close to free fall. Since it is close to free fall, gravity plays a negligible role in stretching this body of fluid and so the dominant force is surface tension. However, the time scale over which surface tension can significantly affect the flow is $s^{1 / 2} / \lambda$ and since $s=O(1)$ in this region, surface tension can only make a significant difference to the shape of the thread over times of order $1 / \lambda \gg 1$. Long before this can occur, the moving transition layer will collide with the boundary and thinning near the boundary as described in Section 5 will occur until the long-wavelength solution eventually ceases to be valid. Thus, over the timescales of interest, this region effectively falls with acceleration close to free fall and its shape is only slightly affected by surface tension.

Therefore, surface tension will remain negligible throughout the entire process if $R \gg \lambda^{2}$. If $R=O\left(\lambda^{2}\right)$, then we would expect surface tension to only play a role with respect to surface-tension-driven pinching in the stationary transition layer. Potentially, surface-tension-driven pinching could occur in this region. Whether pinching will occur will depend on the size of $\lambda$. If $\lambda$ is sufficiently small, the moving transition layer will collide with the boundary and the long-wavelength theory will break down before the surface tension can cause pinching in the stationary transition layer. On the other hand, if $\lambda$ is sufficiently large, the thread will undergo surface-tension-driven pinching in the stationary transition layer. If $R \ll \lambda^{2}$ we would expect surface tension to completely dominate as soon as the stationary transition layer forms. This means that the thread will pinch rapidly after it becomes thin at location $\xi_{s}$ at time $\tau_{s}$.

We now consider case ii in which the thread first becomes thin at the boundary. The solution in this case is given by (62). Far from the boundary, the thread will have $s=O(1)$ and so surface tension must be negligible. We therefore only need to consider the solution in the boundary layer. For $\tau-1=O\left(R^{1 / 2}\right)$, we have $\sigma=O(1)$ and $\sigma_{T}=O(1)$ We immediately see that $s_{\tau} \sim R^{1 / 2} \sigma_{\tau}=\sigma_{T}=O(1)$. On the other hand, the surface tension term $\lambda s^{1 / 2}=O\left(\lambda R^{1 / 4} \sigma\right)=O\left(\lambda R^{1 / 4}\right)$. Since $\lambda \ll 1$ and $R \ll 1$, we conclude that $s_{\tau} \gg \lambda s^{1 / 2}$ and so surface tension is negligible. For $\tau-1 \gg O\left(R^{1 / 2}\right)$, using $(59)$, we have $s_{\tau} \sim \sigma_{T}=O(T \sigma)$. The surface tension term $\lambda s^{1 / 2}=O\left(\lambda R^{1 / 4} \sigma^{1 / 2}\right)$. So surface tension will be negligible if $\sigma \gg \lambda^{2} R^{1 / 2} / T^{2}$. In principle, this condition may be violated. However, for physically realistic parameters, the long-wavelength approximation will typically break down before surface tension becomes important. For example if $\lambda \ll 10^{-1}, R \ll 10^{-2}$ and $T \gg 3$, surface tension would not become important until $\sigma$ was at least as small as $10^{-4}$. Recall that we require $\sigma \gg \delta$ in order for the long-wavelength approximation to hold and so we would require $\delta \ll 10^{-4}$ 
which would represent an extremely slender initial condition. We therefore conclude that in physically realistic situations, surface tension will be negligible whilst the long-wavelength equations remain valid.

\section{Acknowledgment}

The authors would like to thank anonymous referees for their extremely helpful comments and acknowledge financial support from the Research Grants Council of the Hong Kong Special Administrative Region, China [CityU 104211] (JJW), NSERC (HH), and NSF [NSF Award 0709092] (RMM).

[1] Al Kнатів, M.A.M. 2003 The stretching of a viscoplastic thread of liquid. J. Fluids Engg. Trans A.S.M.E. 125, 946-951.

[2] Balmforth, N.J., Dubash, N. \& Slim, A.C. 2010 Extensional dynamics of viscoplastic filaments: II. Drips and bridges. J. Non-Newtonian Fluid Mech. 165, 1147-1160.

[3] Bradshaw-Hajek, B.H., Stokes, Y.M. \& Tuck, E.O. 2004 Computation of extensional fall of slender viscous drops by a one-dimensional Eulerian method. SIAM J. Appl. Math. 67, 1166-1182.

[4] Eggers, J. 2005 Drop formation - an overview. Z. Angew. Math. Mech. 85, 400-410.

[5] Fitt, A.D., Furusawa, K., Monro, T.M. \& Please, C.P. 2001 Modeling the fabrication of hollow fibers: Capillary drawing. J. Lightwave Technologies 19, 1924-1931.

[6] Forest, M.G., Zhou, H. \& Wang, Q. 2000 Thermotropic liquid crystalline polymer fibers. SIAM J. Appl. Math. 60, $1177-1204$.

[7] Green, A.E. 1976 On the non-linear behaviour of fluid jets. Int. J. Engg. Sci. 14, 49-63.

[8] Huang, H., Miura, R.M., Ireland, W. \& Puil, E. 2003 Heat-induced stretching of a glass tube under tension: Application to glass microelectrodes. SIAM J. Appl. Math. 63, 1499-1519.

[9] Huang, H., Wylie, J.J., Miura, R.M. \& Howell, P.D. 2007 On the formation of glass microelectrodes. SIAM J. Appl. Math. 67, 630-666.

[10] Kaye, A. 1991 Convective coordinates and elongational flow. J. Non-Newtonian Fluid Mech. 40, 55-77.

[11] Rodrigues, J.-F. 1987 Obstacle problems in mathematical physics, Holland Mathematics Studies, vol. 134, North-Holland, Amsterdam.

[12] Stokes, Y.M, Bradshaw-Hajek, B.H. \& Tuck, E.O. 2011 Extensional flow at low Reynolds number with surface tension. J. Eng. Math. 70, 321-331.

[13] Stokes, Y.M. \& Tuck, E.O. 2004 The role of inertia in extensional fall of a viscous drop. J. Fluid Mech. 498, 205-225.

[14] Stokes, Y.M., Tuck, E.O. \& Schwartz, L.W. 2000 Extensional fall of a very viscous fluid drop. Q. J. Mech. Appl. Maths. 53, 565-582.

[15] Wilmott, P. 1989 The stretching of a thin viscous inclusion and the drawing of glass sheets. Phys. Fluids A 1, 1098-1103.

[16] Wilson, S.D.R. 1988 The slow dripping of a viscous fluid. J. Fluid Mech., 190, 561-570.

[17] Wylie, J.J. \& Huang, H. 2007 Extensional flows with viscous heating. J. Fluid Mech., 571, 359-370.

[18] Wylie, J.J., Huang, H. \& Miura, R.M. 2011 Stretching of viscous threads at low Reynolds numbers. J. Fluid Mech., 683, 212-234.

[19] Yin, Z. \& Jaluria, Y. 2000 Neck down and thermally induced defects in high-speed optical fiber drawing. ASME J. Heat Transfer 122, 351-362. 


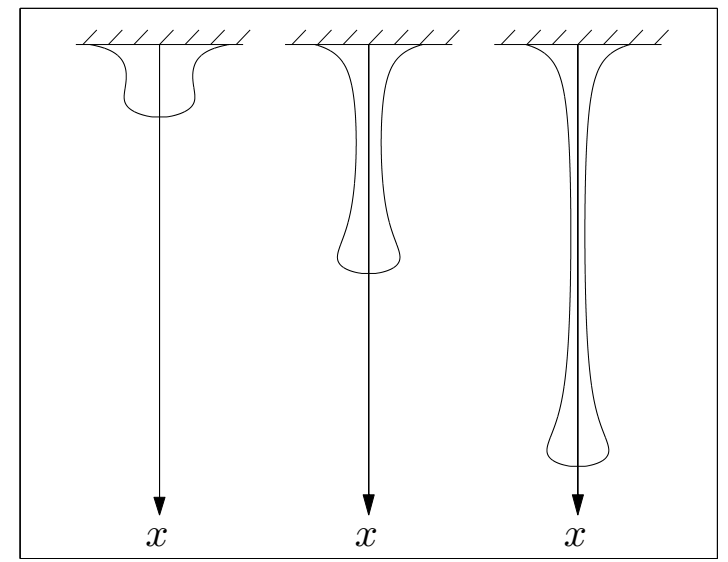

Figure 1: Schematic of a viscous thread extending under gravity. The left panel represents the initial shape of the thread. The other two panels show the thread at two later times.

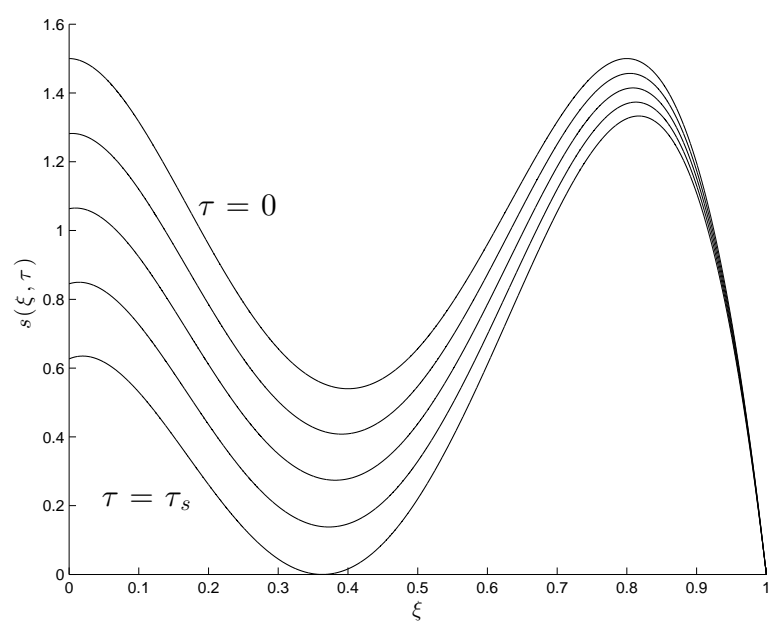

Figure 2: Schematic of the cross-sectional area, $s(\xi, \tau)$, plotted against the Lagrangian variable, $\xi$, at times $\tau=$ $0, \tau_{s} / 4, \tau_{s} / 2,3 \tau_{s} / 4, \tau_{s}$ for $R=0$. The solution breaks down at location $\xi=\xi_{s}$ at time $\tau=\tau_{s}$. 


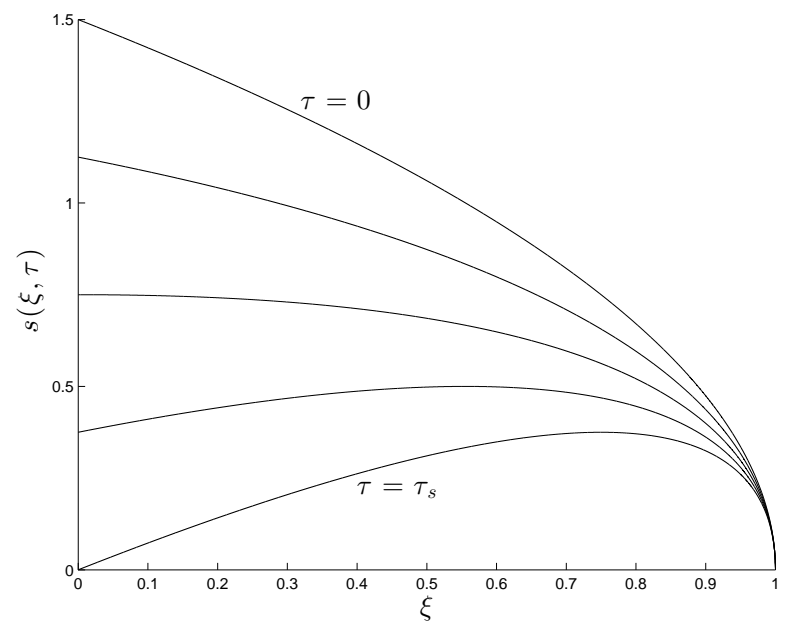

Figure 3: Schematic of the cross-sectional area, $s(\xi, \tau)$, plotted against the Lagrangian variable, $\xi$, at times $\tau=$ $0, \tau_{s} / 4, \tau_{s} / 2,3 \tau_{s} / 4, \tau_{s}$ for $R=0$. The solution breaks down at the boundary $(\xi=0)$ at time $\tau=s_{0}(0)$.

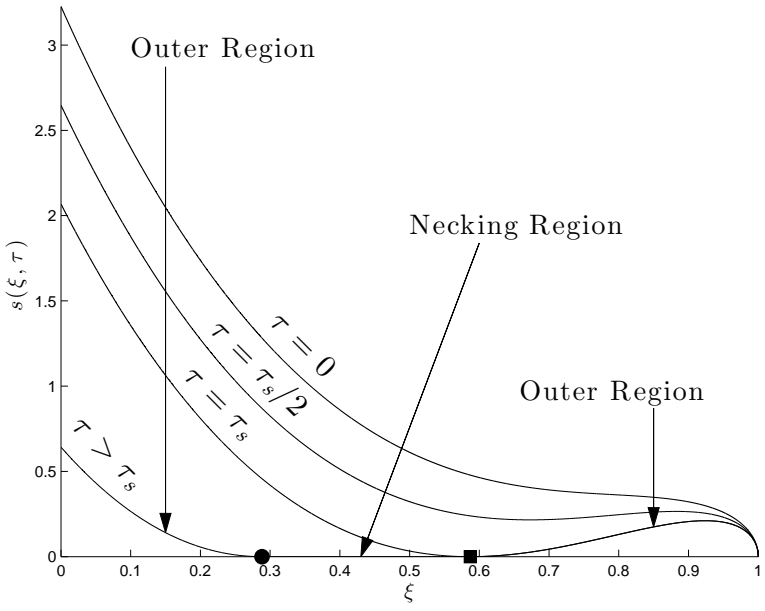

(a)

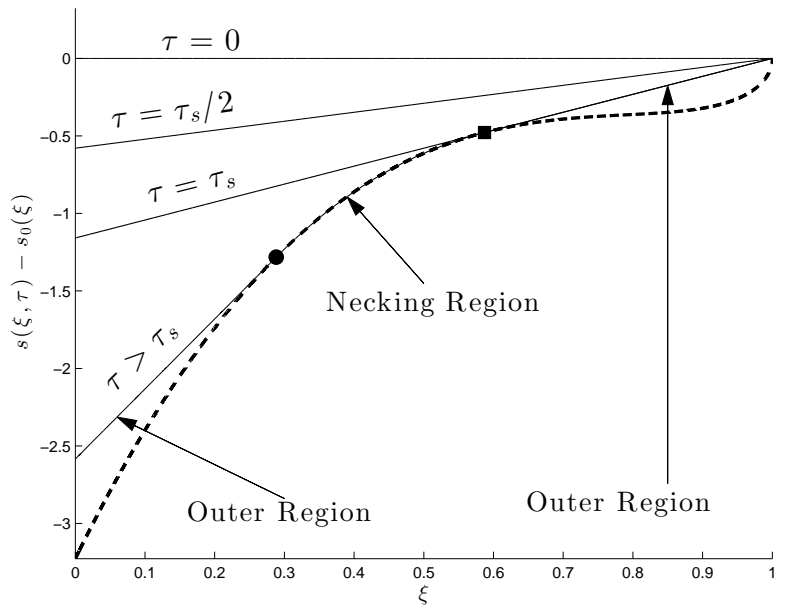

(b)

Figure 4: a) The cross-sectional area, $s(\xi, \tau)$ is plotted against the Lagrangian variable, $\xi$ for a thread with initial shape given by $s_{0}(\xi)=945 \sqrt{1-\xi}\left[1+8(\xi-2 / 3)^{2}\right] / 1334$. The dot represents the moving contact point $\xi_{m}(\tau)$, while the square represents the stationary contact point $\xi_{s}$. For $\tau<\tau_{s}$, inertial effects are negligible throughout the thread. For $\tau>\tau_{s}$, there are two outer regions in which inertial effects are negligible joined together by a thin necking region in which inertial effects are important. b) The data in panel a) is replotted using the deviation from the initial shape $s(\xi, \tau)-s_{0}(\xi)$. The dashed line is $-s_{0}(\xi)$ and represents the value of $s(\xi, \tau)-s_{0}(\xi)$ at which $s(\xi, \tau)$ is zero to leading order. The figure illustrates how finding the contact points $\xi_{m}(\tau)$ and $\xi_{s}$ reduces to a purely geometrical obstacle problem. 


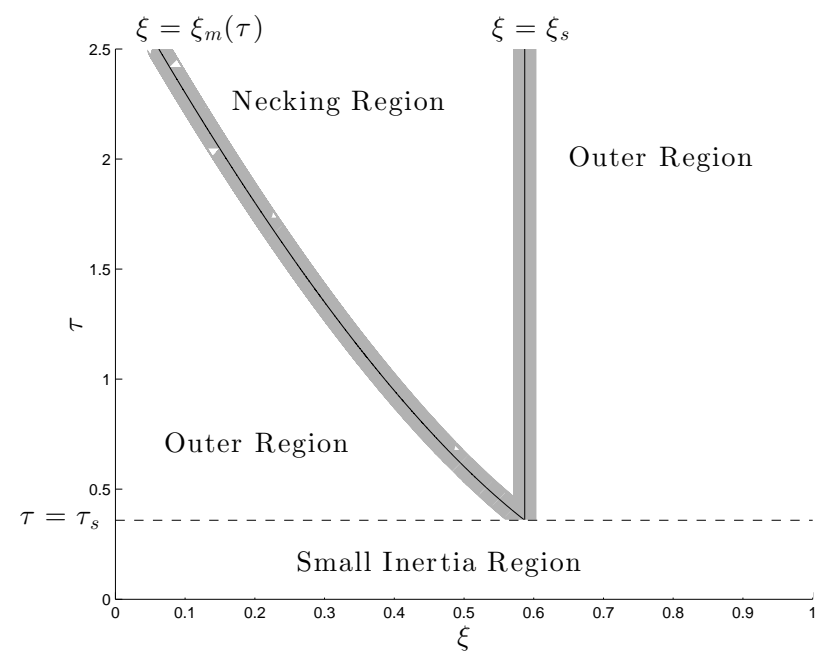

Figure 5: Schematic illustrating the structure of the solution in various regions of the $(\xi, \tau)$-plane for the initial profile shown in Figure 4a. For $\tau<\tau_{s}$, inertia is negligible throughout the thread. For $\tau>\tau_{s}$, there is a necking region, in which inertial effects are important, that connects two outer regions, in which inertia is negligible. There are transition layers (shaded in grey) separating the outer and necking regions.

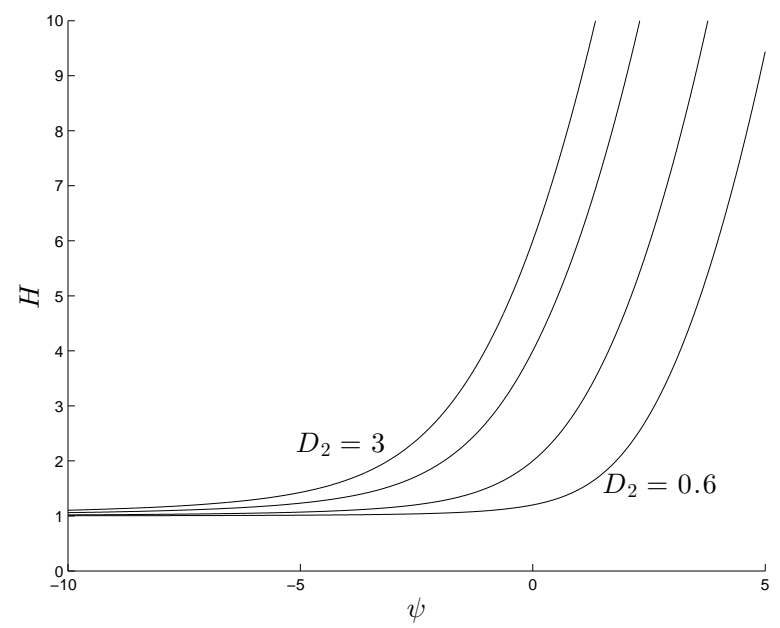

Figure 6: The scaled cross-sectional area $H$ is plotted against the scaled Lagrangian variable for values of $D_{2}=0.6,1,2,3$. 


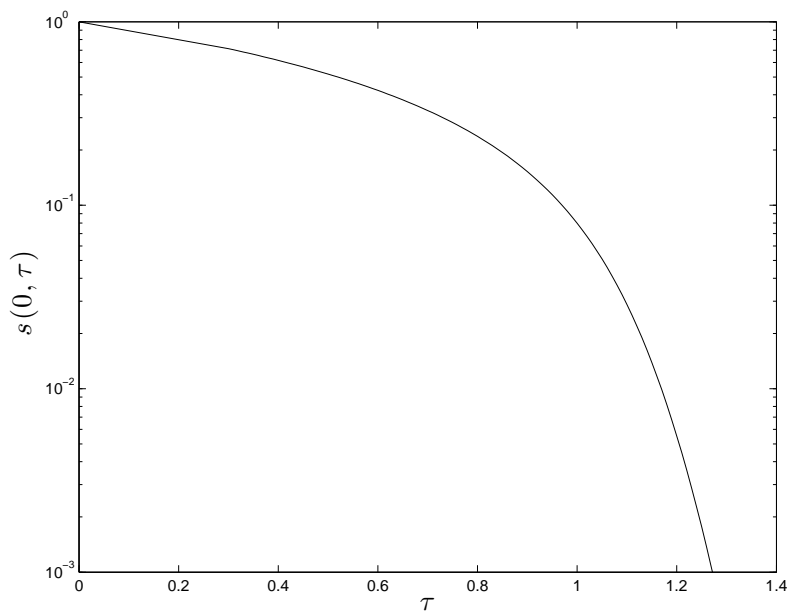

Figure 7: The minimum cross-sectional area is plotted against the time on a semilog scale for $R=10^{-2}$. 